\title{
Evaluation of breeding potential of wild grape originating from Armenia
}

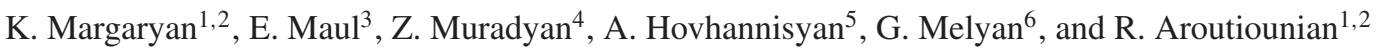 \\ ${ }^{1}$ Department of Genetics and Cytology, Yerevan State University, 1 Alex Manoogian, 0025 Yerevan, Armenia \\ ${ }^{2}$ Research Group of Plant Genetics and Immunology, Institute of Molecular Biology of National Academy of Sciences RA, 7 \\ Hasratyan, 0014 Yerevan, Armenia \\ ${ }^{3}$ Institute for Grapevine Breeding, Julius Kuhn-Institut (JKI), Geilweilerhof, 76833 Siebeldingen, Germany \\ ${ }^{4}$ Department of Plant Growing Product Processing Technology, Armenian National Agrarian University, 74 Teryan, 0009 Yerevan, \\ Armenia \\ ${ }^{5}$ Laboratory of Ethnogenomics, Institute of Molecular Biology of National Academy of Sciences RA, 7 Hasratyan, 0014 Yerevan, \\ Armenia \\ ${ }^{6}$ Scientific Center of Viticulture, Fruit-Growing and Wine-Making of the Armenian National Agrarian University, 1139 Merdzavan, \\ Armenia
}

\begin{abstract}
Crop wild relatives provide a useful source of genetic variation and represent a large pool of genetic diversity for new allelic variation required in breeding programs. Armenia is an important center of origin both for cultivated Vitis vinifera ssp. sativa and wild Vitis vinifera ssp. sylvestris. Owing to recent prospection in Armenian woods and river floodplains many forms of wild grapevine were discovered and inventoried, which is an important prerequisite to unlock their breeding potential in the future. The fact that some genotypes of $V$. sylvestris can withstand the diseases is likely to be due to a more efficient basal immunity. The overall goal of the proposed research was to characterize the diversity of $V$. sylvestris from Armenia with respect to its capacity for stilbene biosynthesis, which might be exploited as genetic resource for resistance breeding. The realized research stimulates the recovery, characterization and preservation of wild grape germplasm, presently at risk of extinction. The recovery and characterization of wild genotypes will be the base of selection of genetic traits important in breeding programs for the generation of biotic and changing climate tolerant grapevine varieties and rootstocks, both necessary for the future of viticulture in Armenia and in Europe.
\end{abstract}

\section{Introduction}

The Caucasus region has been defined by the World Wide Fund for Nature as a Global 200 Ecoregion, based on selection criteria such as species richness, levels of endemism, taxonomic uniqueness, unusual evolutionary phenomena, and global rarity of major habitat types. Moreover, Conservation International has identified the region as a global "hotspot" that is, one of the 25 most biologically rich and most endangered terrestrial ecosystems in the world. The Republic of Armenia, being a relatively small, mountainous country with a total area of $29,740 \mathrm{~km}^{2}$, located at the junction of the biogeographic zones of the Lesser Caucasus and the Iranian and Mediterranean zones, exhibits both, a great range of altitudinal variation and a diversity of climatic zones. While encompassing only 5 percent of the Caucasus area, Armenia is of particular importance as a center of endemism for wild relatives of economically important crops. Crop wild relatives provide a useful source of genetic variation for crop improvement. An overview of 19 different crops revealed that more than $80 \%$ of beneficial traits from crop wild relatives genes were involved in pest and disease resistance [1]. Wild species represent a large pool of genetic diversity for new allelic variation required in breeding programs. The wild grapevine (Vitis vinifera L. subsp. sylvestris Gmel.) is a rare and endangered plant subspecies, heavily threatened in its natural habitats, and high priority given to the collection and preservation of this germplasm is very urgent [2]. Indeed, the preservation of wild populations of Vitis vinifera. ssp. sylvestris is considered essential for the maintenance of genetic variability and the resistance to genetic erosion [3].

Armenia is an important center of origin both for cultivated Vitis vinifera ssp. sativa and wild Vitis vinifera ssp. sylvestris. Besides centuries of tradition in viticulture and winemaking, Armenia possesses a high diversity of local autochthonous, and modern cultivars, as well as wild grape populations. Armenian grape varieties evolved during thousands of years of human selection and their spectrum was further enriched by hybridization. Viticulture is a basic sector of Armenian agriculture and the production of brandy and wines is one of main branches of its export. In the political crisis of the early 1990s, the central collection of Armenia with 22 ha and 850 varieties was destroyed [4]. In the newly established ampelographic collection (Etchmiadzin, Armavir region) about 300 grapevine accessions are preserved including autochthonous, old, long-neglected, endangered grapevine cultivars. Owing to recent prospection in Armenian woods 
and river floodplains many forms of wild grapevine Vitis vinifera ssp. sylvestris (approx. 3000 accessions) were discovered and inventoried, which is an important prerequisite to unlock their breeding potential in the future.

The awareness of the necessity for the protection and conservation of genetic resources of the grapevine wild ancestor Vitis vinifera L. subsp. sylvestris is very high for several reasons. Populations are on the brink of extinction owing to human activities, such as intensive riverbank and forest management; pathogen spread, which has increased in the last decades, and a demanding reproductive strategy. Forest communities with wild grapevine usually do not provide favorable conditions for seed germination and natural gene flow between populations [5]. Many factors constrain the regeneration of wild grapevine, including: scarcity of light; animal grazing; snails feeding on the tender plantlets, and the long distance for pollen to be transmitted between plants in such a context (since the wild grape is diecious, with male and female plants, self pollination is not possible). A particular problem for genetic sustainability of wild grapevine is the presence of American other Vitis species used as rootstocks in vineyards and often escaping, when vineyards are abandoned, as well as cultivated grapevine, which are invasive in the natural habitat [6].

Identification, characterization and unlocking the breeding potential of wild grapes is important to assess genetic diversity and the relationship between wild and cultivated grapes, to understand the domestication of cultivated grapes, and to explore new sources of genetic variation for future grape breeding programs. The present understanding of genetic resources of wild grapevine in Armenia is very limited. Although crucial for documenting grapevine evolution and diversity, the remaining wild grapevine populations are subject to ongoing habitat destruction. The published literature on wild grapevine in Armenia confirms its long presence and noteworthy abundance [7]. So far, no systematic research on wild grapevines from Armenia has been conducted to characterize the population of true $V$. sylvestris and exploitation of grapevine wild relatives for genomicsassisted breeding will have a crucial importance.

During the last years, grapevine wild species have acquired considerable interest, because they represent promising and valuable genetic resources for breeding. Resistance factors from North American wild grapes have been already successfully used to breed new varieties with resistance against Downy and Powdery Mildew, and these varieties have been commercially successful as core element for sustainable viticulture. However, as a strategy to render the success of resistance breeding more sustainable, new sources of resistance are required. Here, the Eurasian ssp. sylvestris, but also wild species from China are attractive resource. For instance, several genotypes of $V$. vinifera ssp. sylvestris show good tolerance against several grapevine diseases, such as Downy Mildew (caused by Plasmopara viticola), Powdery Mildew (caused by Erysiphe necator), and Black Rot (caused by Guignardia bidwelli), which were all introduced only 150 years ago from North America [8]. However, already now, new pathogen strains are encountered that are able to overcome introgressed resistance.This accentuates the necessity to find novel sources of resistance that can be used for breeding. Vitis vinifera spp. sylvestris, as ancestor of cultivated grapevine, and closest living relative, are generally characterised by a robust basal immunity that might be exploited for a new generation of disease resistant grapes. As mentioned above, an important element for sustainable viticulture is resistance breeding using resistance factors originating from wild grapes [9]. These resistance factors have to be understood in context with the complex evolution of plant immunity, which is composed of two levels: an evolutionarily ancient basal immunity, complemented by a more efficient and specific second line of defense. This specific immunity has evolved during a long arms race between pathogen and host plant. Since cultivated grapevine ( $V$. vinifera $\mathrm{ssp}$. vinifera) did not evolve together with these recently introduced pathogens, it represents a native host and in contrast to North American wild species of Vitis, lacks the efficient second layer of innate immunity against these diseases. The fact that some genotypes of $V$. sylvestris can withstand these diseases is likely to be due to a more efficient basal immunity. Since phytoalexins, such as the stilbenes, are the central element of basal immunity, the overall goal of the proposed research is to characterize the diversity of $V$. sylvestris from Armenia with respect to its capacity for stilbene biosynthesis, which might be exploited as a genetic resource for resistance breeding. To achieve this goal it is necessary completely to screen the wild species originated from Armenia to unlock and explore the gene pool of promising wild genotypes.

\section{Material and methods}

\subsection{Plant material}

The wild grapes (Vitis vinifera ssp. Sylvestris, 77 accessions) were collected from mountainous areas and riverbank forests in the Syunik region, the southernmost province of Armenia, 50-60 km away from the villages. Each population was mapped by a GPS receiver. It should be underlined, that in this area as grape growing region in Armenia, the traditional viticulture is performing without grafting and the likelihood of any rootstock gene pool introgression practically is nil.

\subsection{DNA extraction and SSR analysis}

Genomic DNA was extracted from $100 \mathrm{mg}$ of young leaf using peqGOLD Plant Mini Kit according to the manufacturer's protocol (peqLab, Germany). DNA concentration and quality were checked by spectrophotometric analysis and electrophoresis in 1\% agarose gel. Genotyping was carried out by amplifying 25 microsatellite markers (nSSRs). The amplifications were performed as reported in [10]. The amplified loci were detected on an automated ABI $3130 x 1$ Genetic Analyzer (Applied Biosystems, Germany). Allele sizes were scored using GeneMapper 4.0 software (Applied Biosystems, Life Technologies) and recorded in base pairs.

Data analysis. The number of alleles $(\mathrm{Na})$, observed heterozygosity (Ho), expected heterozygosity ( $\mathrm{He})$ and polymorphism information content (PIC) were calculated for 77 wild grapevine accessions using Cervus software version 3.0.7 and GenAlEx softwares [11]. 


\subsection{Leaf sample preparation}

For the analysis the fourth and fifth leaves from the apex, were excised from the grape accessions, and subjected to UV-C stress following incubation upside down on moist filter paper in Petri dishes. For the UV-C treatment, the abaxial surface of the entire leaf were exposed to UV-C light $(254 \mathrm{~nm}, 15 \mathrm{~W})$ for $10 \mathrm{~min}$ at a distance of $12.5 \mathrm{~cm}$. The leaves of the different genotypes were collected at different time points after the treatment, immediately frozen in liquid nitrogen, and stored at $-80^{\circ} \mathrm{C}$ until RNA analysis.

\subsection{RNA extraction and cDNA synthesis}

For RNA extraction, leaves from plants, cultivated at a temperature of $22^{\circ} \mathrm{C}$ and $18^{\circ} \mathrm{C}$ (day and night, respectively) and a photoperiod of $14 \mathrm{~h}$ light and $10 \mathrm{~h}$ dark, were used.

The leaves of selected wild genotypes were harvested at 0 and $2 \mathrm{~h}$ after irradiation, including also non treated controls. Total RNA was isolated using Spectrum ${ }^{\mathrm{TM}}$ Plant Total RNA Kit (Sigma, Deisenhofen) according to the manufacturer's protocol. The extracted RNA was transcribed into cDNA as described by Ismail et al. [12]. The amount of RNA template was $1 \mu \mathrm{g}$.

\subsubsection{Semi-quantitative $R T-P C R$}

Semi-quantitative reverse transcription PCR was performed following 30 cycles of $30 \mathrm{~s}$ denaturation at $94^{\circ} \mathrm{C}$, $30 \mathrm{~s}$ annealing at $60^{\circ} \mathrm{C}$, and $1 \mathrm{~min}$ synthesis at $68^{\circ} \mathrm{C}$ in a conventional PCR according to the Chang and Nick, 2012, [13] using elongation factor-1 $\alpha$ (EF1- $\alpha$ ), (sense, 5'-3' TGTCATGTTGTGTCGTGTCCT; antisense, 5'-3' CCAAAATATCCGGAGTAAAAGA)), resveratrol synthase (RS) (sense, 5'-3' TGGAAGCAACTAGGCATGTG; antisense, 5'-3' GTGGCTTTTTCCCCCTTTAG) and stilbene synthase (StSy) (sense, 5'-3' CCCAATGTGCCCACTTTAAT; antisense, 5'-3' CTGGGTGAGCAATCCAAAAT) primers, performing Taq and ThermoPol buffer (NEB). The PCR products were separated according to Ismail et al., 2012 [12].

\subsubsection{Quantitative real-time $P C R$}

Quantitative real-time PCR was carried out according to Svyatyna et al., 2014 [14]. To compare the mRNA expression level among different samples, the Ct values from each sample was normalized to the value for EF1$\alpha$ as internal standard obtained from the same sample.

This internal standard is widely used in studies on stilbenes due to its stability and reliability and was also found to be very stable in previous studies under different biotic and abiotic stress conditions [12,13]. For each triplicate, these normalized $\mathrm{Ct}$ values were averaged. The difference between the $\mathrm{Ct}$ values of the target gene $X$ and those for the EF1- $\alpha$ reference $R$ were calculated as follows:

$$
\Delta C t(X)=C t(X)-C t(R)
$$

The final result was expressed as $2^{-\Delta C t .(X)}$.

\section{Results and discussion}

Genetic diversity for sylvestris germplasm originating from Armenia: Armenian wild grape V. vinifera ssp.
Table 1. Descriptive statistics and genetic diversity of wild accessions at 25 microsatellite loci.

\begin{tabular}{|c|c|c|c|c|c|}
\hline Locus & $\operatorname{Ra}(b p)$ & $\mathbf{N a}$ & Ho & $\mathrm{He}$ & PIC \\
\hline VVS2 & $121-157$ & 13 & 0.868 & 0.808 & 0.776 \\
\hline VVMD5 & $228-250$ & 10 & 0.816 & 0.834 & 0.808 \\
\hline VVMD7 & $235-263$ & 12 & 0.766 & 0.825 & 0.801 \\
\hline VVMD25 & $239-271$ & 6 & 0.459 & 0.473 & 0.434 \\
\hline VVMD27 & 176-196 & 8 & 0.795 & 0.757 & 0.723 \\
\hline VVMD28 & $228-282$ & 12 & 0.836 & 0.850 & 0.828 \\
\hline VVMD32 & $240-272$ & 11 & 0.883 & 0.845 & 0.822 \\
\hline VrZAG62 & 186-204 & 8 & 0.816 & 0.819 & 0.788 \\
\hline VrZAG79 & $237-261$ & 11 & 0.868 & 0.857 & 0.834 \\
\hline VVIv67 & $348-376$ & 11 & 0.763 & 0.827 & 0.800 \\
\hline VrZAG67 & $121-155$ & 11 & 0.831 & 0.833 & 0.805 \\
\hline VrZAG83 & 188-201 & 4 & 0.800 & 0.704 & 0.642 \\
\hline VVIn16 & $147-155$ & 4 & 0.538 & 0.511 & 0.426 \\
\hline VVIn73 & $260-274$ & 5 & 0.410 & 0.478 & 0.403 \\
\hline VVIp60 & $304-332$ & 10 & 0.770 & 0.774 & 0.745 \\
\hline VVMD24 & $206-218$ & 5 & 0.844 & 0.729 & 0.674 \\
\hline VVMD21 & 244258 & 5 & 0.534 & 0.625 & 0.569 \\
\hline VMC4f3 & $164-214$ & 16 & 0.947 & 0.858 & 0.837 \\
\hline VVIb01 & $293-317$ & 7 & 0.724 & 0.592 & 0.525 \\
\hline VVIh54 & $141-179$ & 11 & 0.842 & 0.814 & 0.785 \\
\hline VVIq52 & $70-86$ & 7 & 0.718 & 0.728 & 0.689 \\
\hline VVIv37 & $150-178$ & 12 & 0.805 & 0.859 & 0.837 \\
\hline VMC1b11 & $167-201$ & 13 & 0.905 & 0.772 & 0.744 \\
\hline ATP3 & $268-466$ & 4 & 0.649 & 0.538 & 0.484 \\
\hline Mean & & 9.00 & 0.758 & 0.739 & 0.702 \\
\hline Total & & 225 & & & \\
\hline
\end{tabular}

Ra: range of allele size (bp), Na: number of alleles, Ho: observed heterozygosity, He: expected heterozygosity, PIC: Polymorphism information content.

sylvestris have not been studied before. The presented research is the part of a large-scale research realized in the scope of Armenian-German bilateral and DAAD projects aimed to evaluate wild grape breeding potential, including analysis of genetic diversity. The molecular fingerprinting of wild accessions was carried out by 25 simple sequence repeat markers encompassing the nine SSR markers recommended by the European project GrapeGen06. Genetic diversity of wild accessions was analyzed for nuclear microsatellites by estimating the range of allele size $(R a, b p)$, average number of observed alleles per locus $(\mathrm{Na})$, observed heterozygosity $(\mathrm{Ho})$, estimated heterozyosity $(\mathrm{He})$ and polymorphism information content $(P I C)$. Statistics about the discriminatory efficiency of the used 25 SSRs markers are presented in Table 1.

The high degree of observed genetic variability among the 77 wild accessions is proven by the high number of different alleles (225). The number of alleles per SSR locus ranged from 4 (VrZAG83, VVIn16, ATP3) to 16 (VMC4f3) and the mean allele number per locus was 9.0. For microsatellite markers efficiency were considered observed and expected heterozygosity ( $\mathrm{Ho}$, $\mathrm{He}$ ) to evaluate the genetic variability among analyzed accessions. Among analyzed accessions the mean value of $\mathrm{Ho}$ was higher than the $\mathrm{He}$, which can be correlated with the outbreeding mating system of dioecious species 


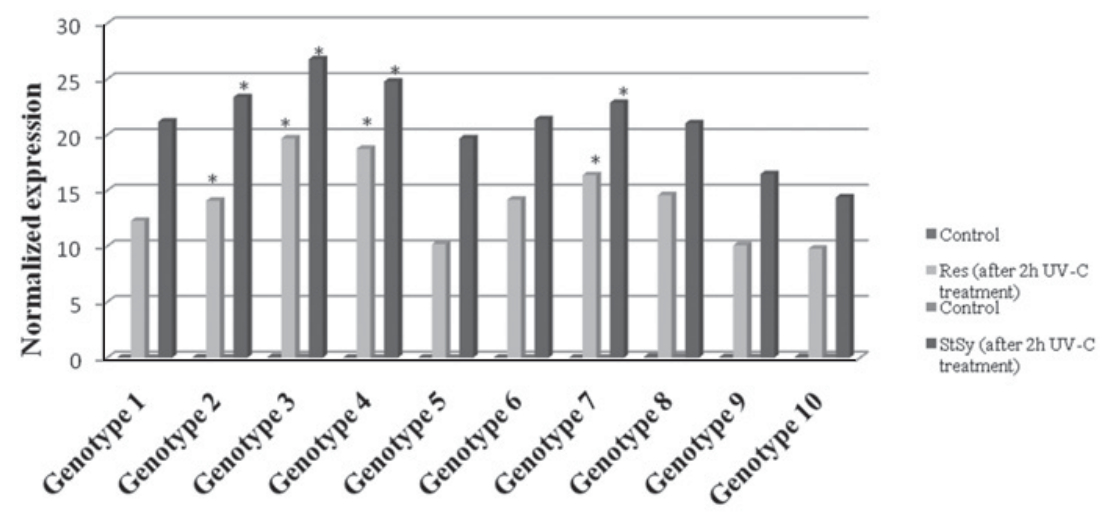

Figure 1. Quantification of transcripts by quantitative real-time PCR normalized to the expression of elongation factor EF1- $\alpha$. *indicates statistically significant difference at the $P<0.05$ level. Data represent mean values from three independent experiments.

including $V$. vinifera $[15,16]$. As an outbreeding species, grapevine possesses considerably heterozygous cultivars affected from severe inbreeding depression.

However as it is shown in Table 1, for the 11 loci from 25 (VVMD5, VVMD7, VVMD25, VVMD28, VRZAG62, VVIv67, VrZAG67, VVIn73, VVIp60, VVMD21, VVIv37) analyzed the $H o$ was slightly lower than $H e$, ranged from 0.410 (VVIn73) to 0.836 (VVMD28) accordingly. This probable can be related with inbreeding or common origin and clonal propagation among the analyzed samples. Obtained results demonstrated, that the expected heterozygosity $(\mathrm{He})$ among analyzed accessions varied within a range between 0.473 (VVMD25) and 0.859 (VVIv37) indicating a high level of genetic diversity within the studied germplasm.

The value of polymorphism information content (PIC) estimates the usefulness of each microsatellite marker for reliable distinction. The calculated PIC values ranged from 0.403 (VVIn73) to 0.837 (VMC4f3) and classified the following loci (VrZAG79, VVMD 28, VVMD32, VVIp31 and VVMD5) as highly informative markers used in research. For cultivar distinction the value of estimated $P I C$ is as indicator of SSRs effectiveness [17].

Stilbene-related genes response to UV-C: Stilbenes represents a small family of phenylpropanoids occurring in plant families, including Vitaceae. As a class of plant secondary metabolites, stilbenes are synthesized inducibly or enhanced constitutively which confer a selective advantage in the defense of plants against biotic and abiotic stresses. The accumulation of stilbenes can be induced by factors such as pathogen infection, wounding, salt stress and UV irradiation. It was revealed that in plants, including grapevine, under normal conditions stilbenes are present only at very low level and the accumulation is starting in response to a wide range of biotic and abiotic stresses as a result of an increased transcription of their biosynthetic genes and the coordinated activation of upstream genes belonging to the general phenylpropanoid pathway [18]. In accordance to the stilbene biosynthetic pathway the general activation of the phenylpropanoid pathway is monitored by probing PAL (phenylalanine ammonium lyase), the stilbene branch of the pathway by probing for StSy (stilbene synthase) and Rs (resveratrol synthase). To analyze stilbene inducibility on a comparative scale, a pulse of UV-C was used as a reliable and standardized input. As it was described the stilbene synthase family in grapevine is extremely expanded, with numerous similar members even in their open reading frames, but differ with respect to their their promoters. Transcripts with partially different expression patterns identified by the StSy and Rs oligonucleotide primers are therefore likely to stem from different members of this family.

According to the obtained results of semi-quantitative reverse transcription PCR no transcripts were detected for the controls independent of the genotype, pointing out that the basal levels of these genes are very low. Among all analyzed samples 10 of them were shown induction of Rs and StSy transcripts after $2 \mathrm{~h}$ of UV-C pulse. The induction of StSy transcripts was accompanied by almost simultaneous induction of Rs transcripts. Analyzed patterns then were examined by quantitative real-time PCR in the same genotypes (Fig. 1). Among tested genotypes under control conditions no significant transcript accumulations were detected for both Rs and StSy genes. Already after $2 \mathrm{~h}$, these transcripts had been clearly induced, with the response of genotypes No 2, 3, 4 and 7 being stronger than other analyzed accessions. In all genotypes, the StSy transcripts increased more strongly and more rapidly in comparison with RS transcripts. Genotype-dependent differences in accumulation of StSy and Rs transcripts among analysed wild grape accessions were observed.

\section{Conclusions}

The main objective of presented study was to explore the pattern of genetic diversity and potential of $V$. sylvestris as a genetic resource for resistance breeding. Our study is the first analysis of the wild grapevines in the southern region of Armenia. During the field prospection, we observed a large number of wild grapevine populations in this area. The subspecies sylvestris exhibited high levels of polymorphism and heterozygosity across the 25 microsatellite loci and its significant diversity was observed. As it was expected the higher levels of heterozygosity in sylvestris was found, in general, because of its obligate out-crossing nature compared to its domesticated counterpart sativa. Based on the observed allele richness this region can be supposed as the centre of diversity for sylvestris. 
The present study demonstrates that genetic factors enabling strong stilbene inducibility are still present in $V$. sylvestris, and might be reintroduced into cultivated grapes. Among the analysed accessions high levels of stilbenes accumulation in response to a UV-C pulse were observed while in control conditions very low basal levels of stilbenes were detected. This means that these genotypes produce their strong induction of stilbenes completely through de novo synthesis. The fact that it is possible to induce stilbene accumulation through an abiotic stress factor revealed the promising possibility that immunity might be activated by appropriate pretreatments with abiotic factors. The induction of tolerance to a certain type of stress by a controlled induction of a different stress pathway is termed "stress priming" and has attracted considerable attention in the context of improving agronomical performance under adverse conditions.

The challenges that face modern agriculture are to develop higher yielding, nutritious and environmentally friendly varieties that improve our quality of life without harnessing additional natural habitats to agricultural production. Armenian sylvestris germplasm are largely unexplored, their identification, conservation and characterization for biotic and abiotic resistance are very important for the future of the vine and wine industry.

The present research was realized as a part of the MES-BMBF project "Breeding potential discovery by multidisciplinary characterization of Armenian grape germplasm", 16GE-042 and DAAD, funding 57378441.

\section{References}

[1] R. Hajjar, T. Hodgkin, Euphytica 156, 1 (2007)

[2] A. Forneck, M. Walker, A. Schreiber, et al., Acta Hort. (ISHS) 603, 549 (2003)
[3] J. Cunha, T. Santos, C. Carneiro, et al., Genet. Resour. Crop Evol. doi: 10.1007/s10722-009-9416-4 (2009)

[4] G. Melyan, S. Gasparyan, Caucasus and Northern Black Sea Region Ampelography, Vitis (special issue) (2012), p. 39

[5] M. Di Vecchi Staraz, Inventory and Characterization of Autochthonous Genetic Resources of Vitis Vinifera L. Subsp. Sylvestris (Gmelin) Hegi in Europe. (Montpellier, France, SupAgro, 2007)

[6] N. Arrigo, C. Arnold, PLos One 2 521e (2007)

[7] Р.А. Ергесян, Виноделие и виноградарство в 6, 9 (1946)

[8] C. Tisch, P. Nick, A. Kortekamp, Proc. 7th Int. Workshop on Gapevine Downy and Powdery Mildew. ISBN: 978-84-7821-827-1 (2014), p. 61

[9] C. Gessler, I. Pertot, M. Perazzolli, Phytopathologia Mediterranea 50, 3 (2011)

[10] T. Lacombe, J.M. Boursiquot, V. Laucou, et al., Am. J. Enol. Viticult. 58, 124 (2007)

[11] S.T. Kalinowski, M.L. Taper, T.C. Marshall, Mol. Ecol. 16, 1099 (2007)

[12] A. Ismail, M. Riemann, P. Nick, J. Exp. Bot. 63, 2127 (2012)

[13] X. Chang, P. Nick, PLoS One 7, e40446 (2012)

[14] M. Kamiya, P. Nick, M. Riemann, Plant, Cell and Environ. 37, 827 (2014)

[15] T. Hvarleva, K. Rusanov, F. Lefort, et al., Vitis. 43, 27 (2004)

[16] T. Dzhambazova, I. Tsvetkov, I. Atanassov, Vitis. 48, 115 (2009)

[17] J.P. Martin, J. Borrego, F. Cabello, J.M. Ortiz, Genome 46, 10 (2003)

[18] D. Duan, D. Halter, R. Baltenweck, C. Tisch, V. Tröster, A. Kortekamp, P. Hugueney, P. Nick, J. Exp. Bot. 66, 3243 (2015) 